räume nicht mit Sicherheit aussagen. Eine longitudinale Periodizität bei der Partikelablagerung im A-Band, wie sie den $\mathrm{Hu}$ x le y schen Vorstellungen über die Spaltung von ATP bei der Verknüpfung und Lösung von Transversalbrücken zwischen Myosin- und Actinfilamenten entspräche, konnten wir niemals auch nur andeutungsweise beobachten.

Ob die in der Z-Scheibe lokalisierte ATPase einem biochemisch bereits bekannten und definierten Enzym entspricht und welche Funktion sie erfüllt, läßt sich zur Zeit noch nicht entscheiden. Es sei jedoch ausdrücklich darauf hingewiesen, daß sie keine
Besonderheit der hochspezialisierten Insektenmuskeln ist. Auch in den zu Vergleichsuntersuchungen herangezogenen Vertebratenmuskeln hat sich die Spaltung von ATP in der Z-Region neben einer solchen im A-Band aufzeigen lassen.

Anm. b. d. Korr.: Nach der Fertigstellung des Manuskripts erschien eine Arbeit von Tice und BarRnetT ${ }^{10}$, in der mit etwas anderer Methodik ATPase-Aktivität in den A-Banden isolierter Myofibrillen des Rattenherzmuskels elektronenmikroskopisch demonstriert wird. Die darin geschilderten Befunde gleichen den von uns berichteten in manchen Punkten, weichen aber in anderen davon ab. Wir werden in der ausführlichen Darstellung unserer Untersuchungen darauf eingehen.

10 L. W. Tice u. R. J. Barrnett, J. Cell Biol. 15, 401 [1962].

\title{
NOTIZEN
}

\section{Methacrylsäuremethylester-eisentetracarbonyl und Vinylacetat-eisentetracarbonyl}

Von E. Koerner von Gustorf, M.-J. Jun und G. O. Schenck

Max-Planck-Institut für Kohlenforschung, Abteilung Strahlenchemie, Mülheim a. d. Ruhr

(Z. Naturforschg. 18 b, 503-504 [1963] ; eingegangen am 26. März 1963)

In unserer 1. Mitteilung ${ }^{1}$ über photochemische Umsetzungen mit $\mathrm{Fe}(\mathrm{CO})_{5}$ berichteten wir über die Darstellung beständiger Philodien-Eisentetracarbonyle nach (1).

$$
\mathrm{L}+\mathrm{Fe}(\mathrm{CO})_{5} \underset{\text { in Benzol }}{\stackrel{h v}{\longrightarrow}} \pi-\mathrm{LFe}(\mathrm{CO})_{4}+\mathrm{CO}
$$

$(\mathrm{L}=$ z. B. Maleinsäureanhydrid, Maleinsäuredimethylester, Fumarsäuredimethylester.)

$\mathrm{Zu}$ den gleichen Verbindungen kann man auch auf thermischem Wege gelangen, wie unabhängig von uns von Weiss, Stark, Lancaster und Murdoch ${ }^{2}$ mitgeteilt wurde, indem man nach (2) $\mathrm{Fe}_{2}(\mathrm{CO})_{9}$ bei $40-45^{\circ}$ in Benzol oder Aceton mit den $\pi$-Donatoren L umsetzt. $\mathrm{Fe}_{2}(\mathrm{CO})_{9}$ muß jedoch wiederum photochemisch aus $\mathrm{Fe}(\mathrm{CO})_{5}$ hergestellt werden ${ }^{3}$.

$$
\mathrm{L}+\mathrm{Fe}_{2}(\mathrm{CO})_{9} \longrightarrow \pi-\mathrm{LFe}(\mathrm{CO})_{4}+\mathrm{Fe}(\mathrm{CO})_{5} \text {. }
$$

Gegenüber der thermischen Arbeitsweise besitzt unser photochemisches Verfahren den Vorteil, daß sich die Umsetzungen mit den $\pi$-Donatoren auch bei tiefen Temperaturen ausführen lassen, so daß sich auch thermisch empfindliche Eisentetracarbonyl-Komplexe dar-

1 G. O. Schenck, E. Koerner von Gustorf u. M.-J. Jun, Tetrahedron Letters 1962, 1059.

2 E. Weiss, K. Stark, J. E. Lancaster u. H. D. Murdoch, Helv. chim. Acta 46, 288 [1963]. stellen lassen. So erhielten wir z. B. durch Belichten von $\mathrm{Fe}(\mathrm{CO})_{5}$ in Methacrylsäuremethylester (I) in 54-proz. Ausbeute nach (3) das Methacrylsäuremethylester-eisentetracarbonyl (II) als orangerote, i. V. unzersetzt destillierbare Flüssigkeit. II ist bei Raumtemperatur unter Argon nur einige Stdn. beständig, bei $-20^{\circ}$ bereits mehrere Tage.<smiles>COC(=O)C(C)C=C(C)C(C)=O</smiles><smiles>COC(=O)[C@H]1[C@@H](C(=O)O)[C@H]1C(=O)O</smiles>

Das thermische Verfahren (2) ist nach Angaben von Werss u. a. ${ }^{2}$ für die Darstellung von II nicht geeignet.

Die in II angenommene Bindung der $\mathrm{Fe}(\mathrm{CO})_{4-}$ Gruppe durch die $\pi$-Elektronen der $\mathrm{C}=\mathrm{C}$-Doppelbindung gründet sich auf folgende Befunde:

HCl spaltet II „hydrierend“ zu Isobuttersäuremethylester (gaschromatographisch identifiziert; $11 \% \mathrm{~d}$. Th.) und $\mathrm{FeCl}_{2}$. Das NMR-Spektrum von II zeigt eine erhebliche chemische Verschiebung der Doppelbindungsprotonen-Signale zu höheren Feldern $\left(\Delta \tau \mathrm{H}_{(\mathrm{a})} \approx+3,2\right.$; $\left.\Delta \tau \mathrm{H}_{(\mathrm{b})} \approx+2,6\right)$. Nach dem IR-Spektrum ist die $\mathrm{C}=\mathrm{C}$-Valenzschwingung in II $\left(1510 \mathrm{~cm}^{-1}\right)$ gegenüber der in I $\left(1642 \mathrm{~cm}^{-1}\right)$ um $132 \mathrm{~cm}^{-1}$ zu niedrigeren Frequenzen verlagert.

Aus Vinylacetat und $\mathrm{Fe}(\mathrm{CO})_{5}$ konnten wir photochemisch das flüssige, rostrote Vinylacetat-eisentetra-

${ }^{3}$ G. Brauer, Handbuch der präparativen anorganischen Chemie, Bd. 2, 2. Aufl., Enke-Verlag, Stuttgart 1962, S. 1510. 

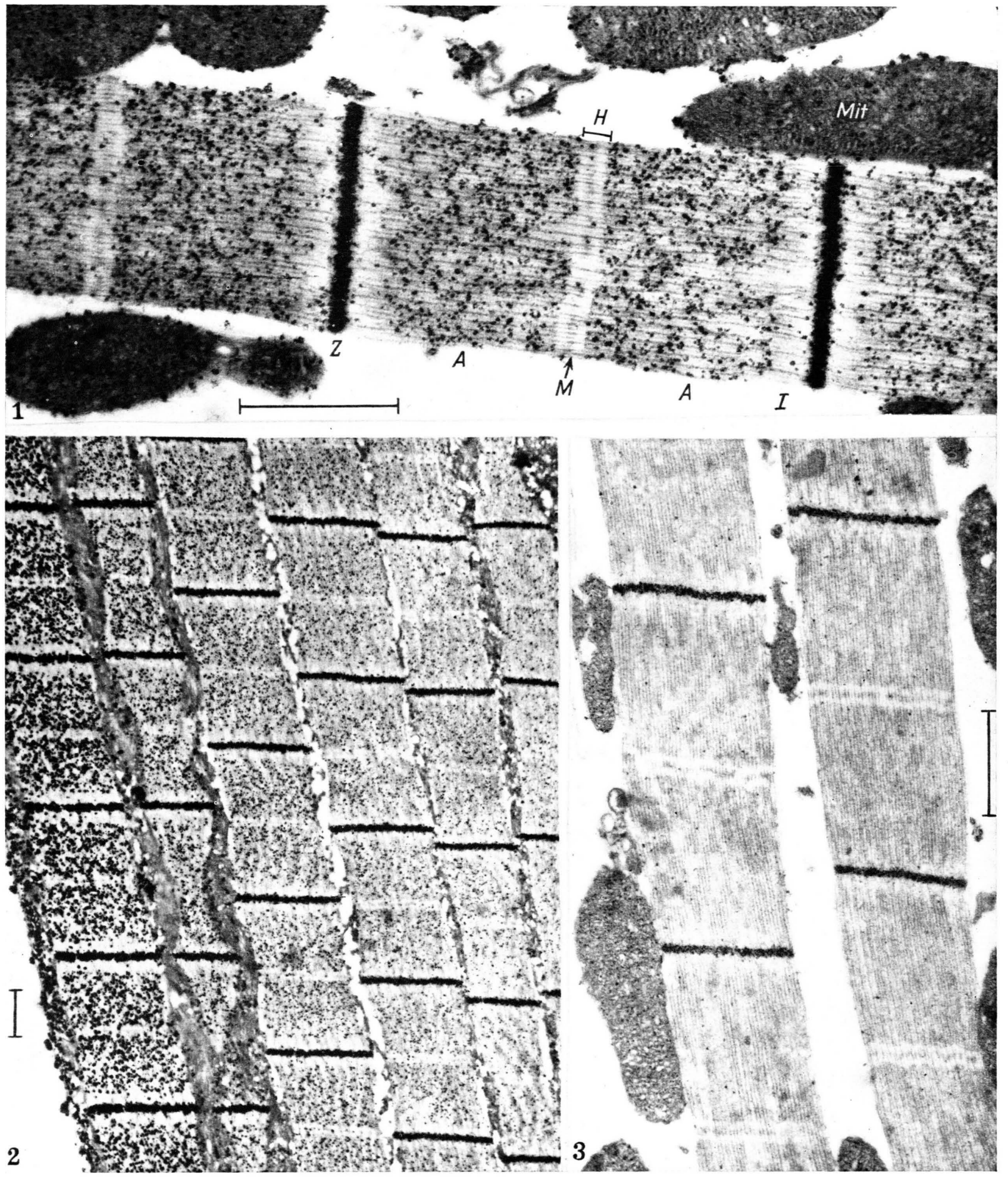

Abb. 1. Phormia-Flugmuskel, Längsschnitt, ATP-Spaltung im

A-Band unter Aussparung der H-Zone und in der Z-Scheibe

Abb. 2. Gleiches Objekt, Übersichtsbild. Starkes Gefälle der sowie auf den Cristae mitochondriales.

Abb. 3. Gleiches Objekt, ATP-Spaltung nur in der Z-Scheibe. Der Maßstab entspricht jeweils $1 \mu$. 

carbonyl (III) gewinnen. III kristallisiert bei ca. $-10^{\circ}$ und ist i. V. unzersetzt destillierbar. Die Aufnahme des IR- und NMR-Spektrums von III wird durch seine Zersetzlichkeit erschwert; auf eine zu $\left[\mathrm{Fe}(\mathrm{CO})_{4}\right]_{3}$ führende ungewöhnliche Spaltreaktion werden wir in anderem Zusammenhang eingehen.

\section{Darstellung}

Meth a c ry ls äuremethy lest e r-eisente tra carbonyl $\quad$ (II): $3,099 \mathrm{~g} \quad(15,8 \mathrm{mMol})$ $\mathrm{Fe}(\mathrm{CO})_{5}$ wurden in $100 \mathrm{ml}$ unter Argon destilliertem I bei $15^{\circ}$ mit einem Quecksilberdampf-Hochdruckbrenner Philips HP $125 \mathrm{~W}$ (Tauchlampenanordnung ${ }^{4}$ ) bis zur Entwicklung von $16 \mathrm{mMol} \mathrm{CO}$ bestrahlt. Überschüssiges I wurde im Wasserstrahlvakuum bei max. $25^{\circ}$ abgezogen, danach gingen bei $10^{-3}$ Torr $2,27 \mathrm{~g}$ $(8,5 \mathrm{mMol})=54 \% \mathrm{~d}$. Th. II über. Sämtliche Operationen wurden unter Argon ausgeführt.

${ }^{4}$ G. O. Schenck, in: A. Schönberg, Präparative organische Photochemie, Springer-Verlag, Berlin 1958, S. 210-234.

\section{Über Aromatenkomplexe von Metallen LXXI ${ }^{1}$}

\section{Cyclopentadienyl-benzol-mono-kohlenoxyd- Kationkomplexe des Molybdäns und Wolframs}

Von Ernst Otto Fischer und Friedrich Johannes Kohl

Institut für Anorganische Chmie der Universität München

(Z. Naturforschg. 18 b, $504-506$ [1963] ; eingegangen am 25. März 1963)

Seit einigen Jahren beschäftigen wir uns mit der Darstellung gemischter Ring-metall- $\pi$-komplexe wie z. B. $\mathrm{C}_{5} \mathrm{H}_{5} \mathrm{CrC}_{6} \mathrm{H}_{6}{ }^{2}$ oder $\mathrm{C}_{5} \mathrm{H}_{5} \mathrm{CrC}_{7} \mathrm{H}_{7}{ }^{3}$. Neuerdings versuchen wir auch Komplexverbindungen zu erhalten, die neben zwei verschieden großen aromatischen Ringen noch weitere Liganden wie $\mathrm{CO}, \mathrm{NO}$ u. a. enthalten. Verbindungen dieses Typs sind bis jetzt noch nicht bekannt. Die Edelgasregel läßt erwarten, daß komplexe Kationen wie $\left[\mathrm{C}_{5} \mathrm{H}_{5} \mathrm{MeC}_{6} \mathrm{H}_{6}(\mathrm{CO})\right]^{\oplus}(\mathrm{Me}=\mathrm{Cr}, \mathrm{Mo}, \mathrm{W})$ stabil sein sollten. Im folgenden wird über ihre Darstellung kurz berichtet.

Zur Synthese des neuen Verbindungstyps schien uns die Umsetzung (1) geeignet.

$$
\begin{aligned}
\mathrm{C}_{5} \mathrm{H}_{5} \mathrm{Me}(\mathrm{CO}){ }_{3} \mathrm{Cl}+\mathrm{C}_{6} \mathrm{H}_{6} \stackrel{\mathrm{AlCl}_{3}}{\mathrm{Benzol}} \\
\quad\left[\mathrm{C}_{5} \mathrm{H}_{5} \mathrm{MeC}_{6} \mathrm{H}_{6}(\mathrm{CO})\right]^{\oplus} \mathrm{AlCl}_{4}{ }^{\ominus}+2 \mathrm{CO} .
\end{aligned}
$$

1 LXX. Mitt.: E. O. Fischer, H. Schuster-Woldan u. K. BittLER, Z. Naturforschg., im Erscheinen.

2 E. O. Fischer u. H. P. Kögler, Z. Naturforschg. 13 b, 197 [1958].

3 E. O. Fischer u. S. Breitschaft, Angew. Chem. 75, 94 [1963].

4 E. O. Fischer u. W. Hafner, Z. anorg. allg. Chem. 286, 146 [1956] ; E. O. Fischer u. J. Seeholzer, Z. anorg. allg. Chem. 312,244 [1961].
Analyse von $\mathrm{C}_{5} \mathrm{H}_{8} \mathrm{O}_{2} \mathrm{Fe}(\mathrm{CO})_{4} \quad(268,0)$

Ber. C 40,33 H 3,01 Fe 20,84,

Gef. C 39,31 H 3,09 Fe 21,12.

Mol.-Gew. (kryosk. in Benzol) : 241.

II ist in Methanol oder Benzol leicht löslich.

Viny lace tat - e is en te tra carbonyl (III) : $2,874 \mathrm{~g}(14,7 \mathrm{mMol}) \mathrm{Fe}(\mathrm{CO})_{5}$ in $100 \mathrm{ml}$ Vinylacetat wurden bis zur Abspaltung von 14,5 mMol CO belichtet. Aufarbeitung in der für II beschriebenen Weise ergab $1,796 \mathrm{~g}(7,1 \mathrm{mMol})=49 \% \mathrm{~d}$. Th. III.

\section{Analyse von $\mathrm{C}_{4} \mathrm{H}_{6} \mathrm{O}_{2} \mathrm{Fe}(\mathrm{CO})_{4}(254,0)$ \\ Ber. C 37,83 H 2,38 Fe 21,99, \\ Gef. C 37,38 H 2,45 Fe 21,80. \\ Mol.-Gew. (kryosk. in Benzol) : 219; 230.}

III ist in Methanol oder Benzol leicht löslich; die Lösungen lassen sich unter Argon bei tiefen Temperaturen unzersetzt halten.

Der Badischen Anilin - und Sodafabrik, insbesondere Herrn Prof. Dr. Sternhofer, danken wir für Chemikalienspenden.

Das Al-Halogenid sollte, wie bei der Dibenzolchromsynthese ${ }^{4}$, zur Auflagerung des Sechsringaromaten auf das Übergangsmetall und als Halogenacceptor ${ }^{5}$ wirken. Im Gegensatz zur schon früher erfolgten Darstellung von

$\left[\mathrm{C}_{5} \mathrm{H}_{5} \mathrm{FeC}_{6} \mathrm{H}_{6}\right]^{\oplus 6}$ aus $\mathrm{C}_{5} \mathrm{H}_{5} \mathrm{Fe}(\mathrm{CO})_{2} \mathrm{Cl} / \mathrm{AlCl}_{3} / \mathrm{C}_{6} \mathrm{H}_{6}$ ließ die Stellung von $\mathrm{Cr}, \mathrm{Mo}, \mathrm{W}$ im Periodensystem bei Berücksichtigung der „Edelgasregel“ erwarten, daß ein Kohlenoxydligand im Molekül erhalten bliebe.

Aus der Umsetzung von $\mathrm{C}_{5} \mathrm{H}_{5} \mathrm{Mo}(\mathrm{CO})_{3} \mathrm{Cl}$ mit $\mathrm{AlCl}_{3}$ in siedendem Benzol wurde mit $\mathrm{NH}_{4} \mathrm{PF}_{6}$ ein tiefroter Niederschlag erhalten, der sich als ein Gemisch zweier Substanzen erwies. Er enthielt neben dem gewünschten $\left[\mathrm{C}_{5} \mathrm{H}_{5} \mathrm{MoC}_{6} \mathrm{H}_{6}(\mathrm{CO})\right]^{\oplus}$ unerwarteterweise noch das gelbe $\left[\mathrm{C}_{5} \mathrm{H}_{5} \mathrm{Mo}(\mathrm{CO})_{4}\right]^{\oplus}$-Kation ${ }^{7}$. Die Bildung des Tetracarbonyls dürfte wohl dadurch zu erklären sein, daß das im Verlauf der oben beschriebenen Umsetzung entbundene CO in einer Konkurrenzreaktion mit dem Ausgangsprodukt nach (2) zu reagieren vermag.

$$
\begin{aligned}
\mathrm{C}_{5} \mathrm{H}_{5} \mathrm{Mo}(\mathrm{CO})_{3} \mathrm{Cl}+\mathrm{CO} & \stackrel{\mathrm{BlCl}_{3}}{\mathrm{Benzol}} \\
& {\left[\mathrm{C}_{5} \mathrm{H}_{5} \mathrm{Mo}(\mathrm{CO})_{4}\right]^{\oplus} \mathrm{AlCl}_{4}{ }^{\ominus} . }
\end{aligned}
$$

Die Bildung des unerwünschten Nebenprodukts ließ sich leicht unterdrücken, indem die Konzentration an

5 E. O. Fischer u. K. Fichtel, Chem. Ber. 94, 1200 [1961].

6 T. H. Coffield, V. Sandel u. R. D. Closson, J. Amer. chem. Soc. 79, 5826 [1957] ; M. L. H. Green, L. Pratt u. G. WilKinson, J. chem. Soc. [London] 1960, 989.

${ }^{7}$ E. O. Fischer, K. Fichtel u. K. Ö́fele, Chem. Ber. 95, 249 [1962]. 